Aus der Universitäts-Hautklinik in Berlin. (Direktor : Prof. Arndt.)

\title{
Zur Frage der Salvarsandermatitis.
}

Von Dr. Wilhelm Heyn, Assistent der Klinik.

Wenn die Anwendung des für die Behandlung und Heilung der Syphilis so wertvollen Salvarsans zuweilen lebensgefährliche Vergiftungen im Gelolge hat, so darf man sich darüber nicht mit dem: Wort von den "unvermeidlich zu bringeuden Opfern" hinwegtrösterr. Die Folgen sind zu schwer, und besonders ist auch die immer größer werdende Gefahr vorhanden, daß das Salvarsan i ${ }_{1}$ Mißkredit gerät. So ist man denn von jeher bemüht gewesell, die Zusammensetzung der Salvarsanpräparate weiter $\mathrm{zu}$ verbessern und namentlich die Regelı für ihre Anwendung so zu vervollkommnen, daß Unglücksfälle vermieden werden. Die Tendenz ging bei der Mehrzahl der Autoren dalin, erstens die Behandlung vorsichtig mit kleinen Dosen zu beginnen, um der sogenanunten Salvarsanidiosynkrasie aus dem Wege zu gehen, zweiteus die Abstände zwischen den Einspritzungen lang genug zu wählen sowie die Höhe der Dosis und die für eine Kur zu verwendende Gesamtmenge des Mittels so zu begrenzen, daB eine Kumulation und die absolute Ueberdosierung vermieden wurden, und drittens eine genaue Beachtung der absoluten und relativen Kontraindikationen gegen die Salvarsanauwendung zu fordern.

Leider hat es sich aber gezeigt, da $B$ immer wieder Unfälle eintraten, und seit dem Kriege hat sich deren Zahl sogar in beuuruhigender Weise vermehrt. Zum Teil liegt das ja sicherlich an der gegen früher sehr viel größeren Zahl der behandelten Fälle und einer vielfach kritiklosen Anwendung des Mittels sowie an der Verschlechterung des allgemeinen Kräftezustandes der Bevölkerung und auch an einer ungenügenden Befolgung der unerläßlichell, allgemeinen VorsichtsmaBregeln. Gewichtige Grüunde veranlassen ferner nicht wenige objek tive Beurteiler, den Salvarsanpräparaten hinsichtlich ihrer exakten chemischen Zusammensetzung bzw. Reinheit niclit mehr so unbedingt wie früher $z u$ vertrauen. Aber diese Dinge sind nicht allein verantwortlich zu machen, vielmehr ist es in erster Linie erforderlich, daß Klarheit herrscht über die Grundlagen unserer Dosierung, welche sich aus der richtigen Deutung der an den Kranken gemachten Beobachtungen ergeben. Da ist zunächst zu betonen; daB es - ganz abgesehen von dell bereits erwähnten Fälleı von Ueberempfindlichkeit gegen Salvarsan - eine praktisch verwertbare "mittlere Toleranz" und damit eine schematisch gültige Einzel-oder gar Gesamtdosis wirksamer Höhe streng genommen überhaupt nicht gibt. Trotzdem in Lehrbüchern and Abhandlungen auf die bei jeder und selbst bei der vorsichtigsten Behandlungsweise einzuhaltenden Kautelen hingewiesen wird, hat sich allmählich in der Anwendung des Salvarsans vielfach ein verhängnisvoller Schematismus entwickelt. Wir behandeln Individuen, und diese haben gegenüber dem Salvarsan durchaus keine generelle, sondern eine individuelle Toleranz, deren Höhe dem Arzt in jedem einzeluen Falle von vornherein unbekannt ist und die férner bei einem und demselben Patienten wiederum durchaus 
keine konstante Größe darzustellen braucht, sondern nicht selten zeitlichen Schwankungen unterworfen ist. Unsere. Aufgabe muB es demnach sein, bei Anwendung der Salvarsanpräparate derart zu indi vidualisieren, daß wir auch eine relative Intoleranz möglichst in jedem Falle rechtzeitig erkennen und so wenigstens schwerere Schädigungen nach Möglichkeit vermeiden. Die Gefahr der relativen Ueberdosierung wird um so größer, je intensiver die Salvarsandarreichung gestaltet wird. Daraus ergibt sich die Folgerung: Einzelund Gesamtdosis müssen um so niedriger und die Zwischenräume zwischen den Gaben. um so länger gewählt werden, je weniger ein Arzt mangels Erfahrung und Schulung oder infolge Ueberlastung in der Lage ist, die oft nicht leicht zu deutenden Anzeichen drohender Intoleranz früh genug $\mathrm{zu}$ bemerken und richtig $\mathrm{zu} \cdot$ Werten, und je schlechter der allgemeine Gesundheitszustand des jeweiligen Krankenmaterials (Großstadt!) ist

Wir werden Ehrlichs großes Erbe am besten verwalten, wemn wir, wie die Dinge heute liegen, mit größter Sorgfalt darüber wachen, $\mathrm{daB}$ vor allem andern niemandem aus der Salvarsananwendung Schaden erwächst, sei es selbst, daß wir das Endziel der Therapia magna sterilisans dabei, gewiß nicht für immer, aber jedenfalls bis auf weiteres, aus dem Gesichtskreis verschwinden sehen. Jede Salvarsanschädigung ist.die Folge einer relativen Ueberdosierung.

Aber ist es denn überhaupt möglich, das drohende Unheil rechtzeitig zu erkennen? Ueber diesen Punkt sind die Ansichten sehr verschieden. Vielfach wird -behauptet, die schweren Erscheinungen pflegten schlagartig einzusetzen, wie ein "Blitz aus heiterem Himmel“, sie zu vermeiden wäre demnach, da keine Dosierungsmethode gänzlich davor schütze, im Prinzip unmöglich. Solch en Ansch a u ung enjedenfalls insoweit, als die Salvarsandermatitis in Frage kommt - entgegenzutreten, ist der Hauptzweck dieser Zeilen.

Selbst wenn es zuträfe, dab auch der Erfahrene dem Eintritt schwerer Salvarsanzwischenfälle zur Zeit stets hilflos ausgesetzt ist, wäre es m.E. von höchst nachteiliger Wirkung, wenn ein derartig fatalistischer Standpunkt weitere Verbreitung finden würde. Es erscheint vielmehr von der größten Wichtigkeit, den Kreis der absoluten und namentlich auch der relativen Kontraindikationen mehr und mehr zu vervollständigen und durch strengere Fassung der Indikationen das Anwendungsgebiet des Salvarsans auf das notwendige $\mathrm{MaB}$ zu beschränken, sowie ferner besonders die Vorsichtsmaßregeln auszubauen und der ärztlichen Allgemeinheit näher zu bringen, die uns die Vermeidung der Schädigungen erleichtern können. Angesichts der z. Zt. bestehenden tatsächlichen Verhältnisse muß festgestellt werden, daB der Arzt bei der noch vielfach gebräuchlichen, sehr weitgehenden Anwendung des Salvarsans zum mindesten vor seinem eigenen Bewußtsein ebenso schwer mit der unabweisbaren Verantwortung für eine Schädigung durch die Behandlung belastet ist, wie etwa ein Chirurg, der eine das Leben unter Umständen gefährdende Operation ohne Indicatio vitalis vornimmt.

Unter den schweren Salvarsanschädigungen spielen neben dem in seiner Pathogenese noch nicht einwandfrei aufgeklärten sogenannten Salvarsanikterus die universellen Dermatitiden praktisch die wichtigste Rolle. Hinsichtlich der Häufigkeit des Vorkommens stehen sie nach dem lkterus an zweiter Stelle. Und wenn auch ein großer Teil schlieBlich in Genesung ausgeht, so sind doch Todesfälle keineswegs die Ausnahme. Stets aber bedeutet eine derartige Lrkrankung monatelanges, schwerstes und qualvollstes Siechtum und daran anschließend eine meist bis zum Eintritt der Erwerbstähigkeit wiederum Monate beanspruchende Rekonvaleszenz, sodaß der Patient oft neben der gesundheitlichen auch schwere wirtschaftliche Einbuße erleidet.

Wie sieht es nun mit den Vorboten bzw. der eventuellen Vermeidbarkeit solcher schweren Zufälle aus? Darüber geben einige Krankellgeschichten, die ich im Folgenden mitteile, vielleicht einigen Aufschluß. Hervorheben möchte ich, daß es sich bei meinen Fällen durchweg um "reine" Salvarsandermatitis handelt, sodaß also diejenigen Einwände, welche sich bei voraufgegangener kombinierter Behandlung aus der vollkommenen klinischen Analogie der universellen $\mathrm{Hg}$ Dermatitis mit der Hautentzündung durch Salvarsan ergeben, hier ohne weiteres entfallen.

Ich darf mich bei der Schilderung meiner Beobachtungen hinsichtlich der oft und genau beschriebenen dermatologischen Details des im ganzen ja ziemlich einförmigen Krankheitsbildes kurz fassen und werde den Schwerpunkt der Darstellung auf diejenigen Daten der Vorgeschichte verlegen, auf die es im Zusammenhang der vorliegenden Arbeit hauptsächlich ankommt.

1. Fall. F. R., 19jähriger Mann, bekam seiner Angabe nach wegen eines "verdächtigen Geschwürs" innerhalb von vier Wochen 8 Injektionen zu je 0,6 Neosalvarsan. 14 Tage nach der letzten Einspritzung trat ein Ausschlag auf, der sich innerhalb der nächsten Woche so verschlimmerte, daß Patient die Klinik aufsuchen mußte. Bei der Aufnahme am 15.1V. 1920 bestanden eine schwere, universelle, mit Nässen und Krustenbildung einhergehende Dermatitis, hohes Fieber, schwere Prostration, heftige subjektive Beschwerden, völlige Schlaflosigkeit. Die inneren Organe boten keinen krankhaften Befund. Urin frei von Eiweiß und Zucker. Nach drei Wochen waren die akuten Ersch einungen zurückgegangen. Starker universeller Haarausfall. Während der zwei Monate beanspruchenden Rekonvaleszenz bestand noch lange Zeit Rötung und Schuppung. An den Händen, namentlich an den Streckseiten der Finger und an den Handtellern, entwickelte sich eine diffuse Hyperkeratose mäßigen Grades. Ausgang in Heilung.

Wir haben hier also einen Fall vor uns, welcher, falls die Angaben des Patienten zutreffen, aus einer relativen Indikation - die syphiliische Natur des Schankers war nicht sicher festgestellt - eine Abortivkur mit Salvarsan unterzogen wurde. Was die Dosierung anbetrifft, so kann nach meiner Ansicht kaum bestritten werden, dal die Einzelgaben absolut zu hoch, die Zwischenräume zwischen den Einspritzungen (2-3 Tage) absolut zu kurz waren. Aus den Angaben des Krankeıl ergibt sich nichts darüber, daß während der Behandlung Anzeichen schlechter Verträglichkeit (Fieber, Gewichtsverlust) aufgetreten wären. Hervorzuheben ist das späte Einsetzen der Dermatitis, ie erst 14 Tage nach Beendigung der Kur begann.

2. Fall. H. B., 48jähriger Mann, starker Raucher, sehr nervös, aufgenommen am 29. 1. 1921, gibt an, daß am 29. X11. 1920 durch seinen Arzt wegen eines syphilitischen Primäraffekts eine Salvarsankur- eingeleitet worden sei. Bis zum 18.1.1921 sei jeden 2.-3.Tag eine intravenöse Salvarsaneinspritzung (unbekannt, welchen Präparates) gemacht worden. Er habe insgesamt 7 Einspritzungen von je 0,2 bis $0,4 \mathrm{~g}$, im ganzen $2,0 \mathrm{~g}$ des Mittels erhalten. Die Kur sei ihm von Anfang an schlecht bekommen, er habe dauernd, besonders im unmittelbaren Anschlu $B$ an die Einspritzungen, bis über $39^{\circ}$ Fieber gehabt und wiederholt Bedenken geäußert, besonders, als er am 12. I einen Ausschlag zunächst an den Beinen bemerkte, der sich allmählich unter Zunahme des Krankheitsgefühls über den ganzen Körper ausbreitete. Seit 21. I. liegt Patient zu Bett, ist völlig ohne Schlaf, appetitlos, von zunehmenden Beschwerden geplagt. Befund: Schwere universelle exfoliierende, zum Teil hämorrhagische Erythrodermie und Dermatitis. Starke Schwellung, Nässen, Krustenbildung, namentlich der Haut des Gesichts, der Ohrmuscheln, Unterarme, Unterschenkel, Genitalgegend. Schwellung und Rötung auch der Mundschleimhaut, besonders des Zahnfleisches; sehr defekte Zähne, starker Foetor ex oré. An den inneren Organen, abgesehen von einer diffusen Bronchitis, kein, krankhafter Befund. Urin frei von Zucker und Eiweiß Temperatur: $39^{\circ}$. Während der vierwöchigen Beobachtung (Behand lung: Zinktrockenpinselung, Verbände mit weicher Zinkpaste, Bäder) ließen die heftigen Fieberbewegungen allmählich nach, und die Dermatitis bildete sich langsam so weit zurück, daß die immer noch dunkel gerötete Haut größtenteils nur noch trocken schuppte. Gleichzeitig entwickelte sich ein übermächtiger Juckreiz, und infolge des Kratzens traten zahlreiche Furunkel auf. Es bestand anhaltende quälende Schlaflosigkeit, zunehmende Hinfälligkeit. Am 24.11. verließ $\mathrm{Br}$. gegen unsern Rat die Klinik und suchte eine Dermatologische Privatklinik auf, wo er einige Tage später an einer Pneumonie zugrundeging.

Bei der Beurteilung dieser Krankheitsgeschichte wäre zunächst die Frage zu beantworten, ob sich die Schädigung eventuell hütte vermeiden lassen. Und da muß gesagt werden, $\mathrm{daB}$ bei einem 48jährigen Mann, bei dem Alkohol- 1nnd NikotinmiBbrauch vorlag, eine besonders vorsichtige Dosierung (Beginn mit kleinsten Dosen, allmähliche Steigerung, genügende Intervalle zwischen den einzelnen Einspritzungen) am Platze gewesen wäre. Die Zwischenräume zwischen den Salvarsaninjektionen waren auch hier viel zu kurz. Diese ohne Rücksicht auf die relativen Kontraindikationen unternommene Behandlung und unvorsichtige Dosierung wurde trotz ständiger, von Anfang an den Kranken und seine Angehörigen beunruhigender Temperatursteigerungen und sonstiger ungünstiger Allgemeinerscheinungen und trotz mehrfachen Einspruchs von seiten des Patienten Wochen hindurch mit einem geradezu unbegreiflichen Schematismus durchgeführt. Auch durch das Auftreten eines zunächst banal aussehenden, ekzemähnlichen Ausschrlags an den Unterschenkeln in seinem Vorgehen nicht stutzig gemacht, setzte der Arzt seine Einspritzungen so lange fort, bis der Patient durch die mittlerweile universell gewordene Dermatitis bettlägerig geworden und an dem weiteren Erscheinen in der Sprechtunde verhindert war.

3. Fall. K.S., 22jähriger Mann, der im Verdacht stand, seine Braut infiziert zu haben, wurde lediglich, weil die einmal angestellte Wa.R. ein positives Resultat ergeben hatte, ohne dab sonstige Anzeichen von Syphilis vorhanden waren, von privatärztlicher Seite einer Salvarsanbehandlung unterzogen. In der Zeit vom 12. II. bis 7.111. 1921 in Abständen von 2-3 Tagen, bekam Patient 11 Einspritzungen von je 0,2 Sulfoxylatsalvarsan. Nach der 10 . Gabe, am 6 . Ill., bemerkte er einen juckenden Ausschlag an den Händen und zeigte diesen dem Arzt, der ihm trotzdem am 7.11I. die 11. Einspritzung verabfolgte. Am Abend desselben Tages trat hohes Fieber ein. Am 8. III. trat ein Ausschlag am Rumpf auf, der vom Hausarzt, einem Nichtdermatologen, für Scharlach gehalten wurde. Bei der Einlieferung in die Universitätshautklinik am 11. Ill. bot der Kranke, neben einem fast universellen, teilweise noch skarlatiniformen, an den Beinen hämorrhagischen Erythem, an Händen und namentlich Füßen ausgedehnte, bis hühnereigroße Blasen mit teilweise hämorrhagischem lnhalt, solche geringere Größe auch im Gesicht und am Halse dar. Die Schleimhaut der Mundhöhle, insbesondere der Zungenrücken und die Innenflächen der Lippen und Wangen zeigten auf geröteter und geschwollener Basis dichtstehende, stecknadelkopfgroße Bläschen bzw. Erosionen und punktförmige Hämorrhagien. Innere Organe ohne Befund. Temperatur 39,5. Verlauf: Hohes, fast kontinuierliches Fieber, völlige Schlaflosigkeit, große Schmerzhaftigkeit der Haut, besonders im Bereich der Blasen. In den ersten Beobachtungstagen kommt es zur ausgebildeten Dermatitis: namentlich in den Beugen und auch am Rumpf zeigt die Haut ödematöse Durchtränkung, Bläschenbildung. In der Folge gingen die akut entzündlichen Erscheinungen unter Behandlung mit Kalkwasser- 
liniment und Bädern zurïck. Nachdem schon am 16. III. Schmerzen und neuerdings hohes Fieber aufgetreten waren, wurde am 18. III. inmitten einer diffus geschwollenen Hautpartie auf eine walnußgroBe Resistenz in der linken Axilla, die für einen AbszeB gehalten wurde, inzidiert. Bei der Inzision fand sich jedoch kein AbszeB, sondern eine Drüse von trocken nekrotischer Beschaffenheit. An den folgenden Tagen nahm die Schwellung weiter $\mathrm{zu}$, das Allgemeinbefinden verschlechterte sich rasch, eine am 26. III. noch angelegte, breite Inzision in die erkrankte Partie ergab eine tiefgreifende eitrige Infiltration der Weichteile. Eine halbe Stunde später trat der Exitus ein. Alls dem Sektionshefund (Prof. Ce elen): Phlegmone der linken Achselhöhle und Umgebung, beiderseitige eitrige Pleuritis, eitrige Peritonitis, Mediastinalphlegmone, septische Parenchymdegeneration. Bakteriologisch: Hämolytische Streptokokken im plılegmonösen Gewebe und steril entnommenen Venenblut.

Auch in diesem Falle ist der Forderung, eine energische Salvarsanbehandlung nur auf Grund ausreichender Indikation vorzunehmen, nicht genügend Rechnung getragen worden. Die Einzeldosis war zwar nicht übermäBig hoch, jedoch waren auch hier die Zwischenräume (2-3 Tage) viel zu kurz, ganz besonders für Sulfoxylatsalvarsan, das sich bekanntlich durch eine besonders lange Remanenz im Organismus auszeichnet und bei dem also die Gefahr der Kumulation besonders nahe liegt. Auch hier dokumentierte sich das Herannahen der schweren Hauterscheinungen bereits nach der 10 . Einspritzung durch das Auftreten eines geringfügigen, banal aussehenden Ausschlags an den Händen, und wenn auch nicht mit Sicherheit behauptet werden kann, daB durch sofortige Unterbrechung der Kur das Eintreten schwerer Erscheinung'en ganz vermieden worden wäre, so hat doch ohte Frage die unter Nichtbeachtung der erwähnten Warnungszeichen verabreichte 11. Einspritzung noch weiter erheblichen Schaden gestiftet.

4. F all. P. R., 34 Jahre alt, 1908 wegen sekundärer Sypliilis mit mehrwöchiger Schmierkur, 1909-1913 auljerdem viermal mit je 12 Injektionell von Hydrargyrum salicylicum behandelt, erkrankte 1920 mit Drüsenschwellung am linken Kieferwinkel. Wa.R. ++++ . Einleitung einer Schmierkur. Eine Salvarsane inspritzung hat te 14 Tage lang. Abgeschlagenheit und Kopfschmerzen im Gefolge. Weitere Zunahme der Drüsenschwellungen, die auch auf Röntgenbestrahlung nicht wichell. Nunmehr wurden 20 Injektionen von Hydrargyrum salicylicum verabreicht, ohne dab sich die Drüsenveränderungen zurückbildeten. Nach kurzer Pause wurde eine Salvarsankur begonnen. Es wurde wöchentlich eine Einspritzung von $0,6 \mathrm{~g}$ gemacht ${ }^{1}$ ). Nach der 6 . Einspritzung empfand der Patient sofort eill kurz dauerndes Gefühl von Prickeln am ganzen Körper. Zwei Tage später trat eine Anșchwellung des einen Armes und ein juckender Ausschlag hinter den Ohren und an den Fußkı̈cheln auf.- Der Arzt meinte, diese Erscheinungen hätten mit dem Salvarsan nichts zu tun, und machte eine 7. Einspritzung. Nun verschlimmerte sich der Ausschlag rasch und breitete sich unter Fieber, Appetitlosigkeit und quälender Schlaflosigkeit über den ganzen Körper aus. 14 Tage nach der 7. Einspritzung wurde der Kranke am 24. V. aufgenommen. Der große, krättige, wohlgenährte Mann bot eine diffuse, universelle, im Gesicht und an Händen und Füßen mit Exsudation einhtergehende, im übriger. in Rötung, Schwellung und reichlicher Schuppung bestehende Dermatitis dar. Das Allgemeinbefinden war bis auf die quälende Schlaflosigkeit nicht besonders schwer gestört. Der Fall verlief günstig. Als der Kranke Ende Juli als bis auf weiteres arbeitsunfähiger Rekonvaleszent entlassen wurde, bestand noch eine gewisse Spannung und Trockeulheit der Haut. Auf dem behaarten Kopf Schuppenbildung. Handteller und Fußsohlen waren Sitz einer flächenhaften, hochgradigen Hyperkeratose. Am Nacken-, Hals- und Rumpfhaut hatte sich eine schmutzig-graubräunliche, verwaschene Pigmentierung entwickelt.

Mán könnte sich fragen, ob das Vorhandensein einer Drüsenschwellung, deren syphilitische Natur zudem, trotz der positiven Wa.R., keineswegs iber jeden Z Zweifel erhaben war, genügend Anla $\beta^{\circ}$ zur Einleitung einer Salvarsanbehandlung mit derart massiven Dosell sein konnte. Um so melnr; als der Kranke ein ma l a u f e in e Salvarsaneinspritzung mit wochenlanger Störung seines Wohl. befindens reagiert hatte. Der entscheidende Fehler war aber wiederum die AuBerachtlassung der prämonitorischen $\mathrm{H}$ autveränderungen, welche infolge ihres scheinbar banalen Aussehens sehr mit Unrecht für ein akzidentelles Ekzem gehalten wurden. -

In sämtlichen angeführten rantıl traten die schweren Störungen nicht gleich im Anfang, sondern erst einige (2-6) Wochen trach Beginn der Kur auf, sodaß eventuell Gelegenheit gewesen wäre, allerleichteste Anzeichen schlechter Toleranz bei geniigend sorgfältiger Beobachtung der Kranken wahrzunehmen.

Abgesehen vom ersten Fall, ließ sich bei sämtlichen Kranken das Vorhergehen gröberer Störungen nachweisen. Sie hätten zur sofortigen Unterbrechung der Kur Veranlassung geben müssen, wodurch wahrscheinlich der schwere Verlauf vermieden worden wäre.

Die universelle Dermatitis trat sowohl nach Neosalvarsan wie nach Sulfoxylatsalvarsan auf. Gerade in letzter Zeit ist betont worden, $\mathrm{da} B$ bestimmte Präparate, namentlich Silbersalvarsan und Sulfoxylatsalvarsan, vorwiegend Salvarsandermatitis machen und daß im Ver-

1) Trotzdem der Patient vor Einleitung der Salvarsanbehandlung auch $\mathrm{Hg}$ bekommen hatte, ist doch ein $Z$ weifel an der ätiologischen Bedeutung des Sálvarsäns flir die Entstehung der Hautentzlindung nicht angängig, da diese mindestens 6 Wochen nach der letzten $\mathrm{Hg}-$-njektion aufgetreten ist. gleich dazu das Neosalvarsan in dieser Hinsicht mehr oder weniger harmlos sei. Aus meinen Beobachtungen geht hervor, $\mathrm{da} B$ auch nach Anwendung von Neosalvarsan, wie überhaupt wohl $\mathrm{n}$ ach jedem Salvarsanpräparat, die erwähnten schweren $S$ chädi gungen a uftreten können. Daß sie in einem Teil der Fälle vermieden werden können, ist höchst wahrscheinlich und geht auch aus anderen in der Literatur niedergelegten Beobachtungen hervor Und auch in denjenigen Fällen, in denen die Katastrophe schlagartig einzusetzen schien, ist das Voraufgehen leichter Warnungszeicheh natürlich sehr schwer auszuschließen, besonders, wenn es sich um ambulant behandelte Kranke handelt. Temperatursteigerungen, die bei klinisch behandelten Patienten zur Vorsicht mahnen und unter Umständen eine vorübergehende Unterbreclıung oder doch Abschwächung der Kur zur Folge haben würden, können von poliklinischen Kranken sehr leicht übersehen werden, wenn man diese nicht anhält, wie dies sehr wünschenswert wäre, regelmäßig oder doch mindestens am Tage v or jeder Einspritzung und am Tage der Einspritzung selbst ihre Temperatur sorgfältig zu messen. Gewichtsabnahme wird von ambulant behandelten Patienten sehr oft nicht genügend beachtet und doch kann sie der einzige Indikator der schlechten Verträglichkeit sein. Es ist unbedingt erforderlich, das Gewicht mindestens einma in der Woche festzustellen und bei stärkeren Einbußen die Behandlung zu unterbrechen oder zu modifizieren.

Vor allem ist auf starken Kopfschmerz, Erbrechen, Durchfälle sorgfältig zu achten.

In diesem Zusammenhang ist hervorzuheben, daß manche Kranke, in dem an sich sympathischen Bestreben, die Kur, auf deren Wirkung sie große. Hoffnungen setzen, uicht zu stören, ihre Beschwerden hartnäckig verheimlichen.

Jede $\mathrm{H}$ autveränderung, die während der Kur auftritt und bei der auch nur die Möglichkeit eines Zusammenhanges mit der Salvarsanmedikation besteht, ist eine Indikation zur sofortigen Unterbrechung der Kur. Es kann nicht genug betont werden, daß die ersten Erscheinungen von seiten der Haut absolut harmlos und banal aussehen, eine gewöhnliche Dermatitis, ein Ekzem usw. vortäuschen können und daB die Verkennung gerade in solchen Fällen die schwersten Folgen hat. In einem unserer Fälle von Salvarsandermatitis wurde der Beginn der letzteren als Pruritus der Fußsohlen auf Schweißeinwirkung zurückgeführt, in einem andern Fall wurden die ersten ekzemähnlichen Veränderungen an den Fingern als Krätze aufgefaßt. DaB uns die sorgfältigste Beobachtung aller derartigen, im Verlauf einer Salvarsankur auftretenden Veränderungen an die Grenzen dessen führt, was man als krankhafte Störung erkennen kann, ist natürlich kein Gruud dagegen, an der Verfeinerung unserer Beobachtung weiterzuarbeiten, wenn man bedenkt, daB man dadurch unter Umstä̉nden großes Unglück verhuiten kann.

Es ist sehr verhängnisvoll, sich damit abzufinden, daß die Salvarsandermatitis quasi ein notwendiges Uebel wäre, das in einem mehr oder weniger hohen Prozentsatz der behandelten Fälle eintreten müBte. In einer großen Zahl von Fällen ist sie höchstwahrscheinlich durch sorgfältigste Beobachtung der erwähnten Störungen, die als Vorläufer auftreten können, zu vermeiden. 